\title{
EFEITO DA BIOESTIMULAÇÃO, MONENSINA E SOMATOTROPINA RECOMBINANTE BOVINA SOBRE O GANHO MÉDIO DIÁRIO E INÍCIO DA PUBERDADE EM NOVILHAS GIROLANDO CRIADAS A PASTO
}

\section{Effect of biostimulation, monensin and recombinant bovine somatotropin on the daily weight gain and onset of puberty of Girolando heifers raised in pastures}

LIMA, C.S. ${ }^{1}$; GAMBARINI, M.L. ${ }^{2}$; VIU, M.A.O. ${ }^{3}$; FILHO, B.D.O' SANTOS, F.C. ${ }^{1}$; CAIXETA, L.S. ${ }^{4}$

\footnotetext{
${ }^{1}$ Mestrando em Ciência Animal - EV/UFG ;

2 Professor da Escola de Veterinária - UFG/Goiânia;

${ }^{3}$ Professor da Escola de Veterinária - UFG/Jataí, Laboratório de Reprodução;

${ }^{4}$ Graduando em Medicina Veterinária - UFG/Goiânia.
}

\section{RESUMO}

Com o objetivo de avaliar o efeito de três estratégias de manejo sobre a função ovariana de novilhas Girolando criadas a pasto, 80 fêmeas com 10 meses de idade e peso de $169,3 \pm 26,4 \mathrm{~kg}$ foram alocadas em oito grupos e submetidas por 180 dias aos tratamentos: Controle (T1); Bioestimulação (T2); Monensina (T3); Somatotropina Recombinante Bovina - rBST (T4); Bioestimulação e Monensina (T5); Bioestimulação e rBST (T6); Monensina e rBST (T7); Bioestimulação, Monensina e rBST (T8). A atividade estral foi avaliada diariamente e os pesos tomados a cada 15 dias. Entre 15 e 16 meses de idade as fêmeas foram submetidas à ultra-sonografia ovariana, a fim de verificar folículos e corpos lúteos. O ganho médio diário $(\mathrm{kg})$ foi $0,52 \pm 0,02(\mathrm{~T} 1) ; 0,42 \pm 0,03$ (T2);

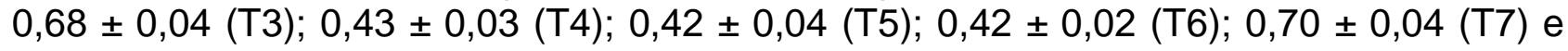
$0,53 \pm 0,01$ (T8), verificando-se diferenças entre eles $(P<0,05)$. O peso aos 16 meses variou de $270 \mathrm{~kg}$ (T2) a $323 \mathrm{~kg}(\mathrm{~T} 3)$, com diferenças $(\mathrm{P}<0,05)$. Os sinais de estro foram observados nas fêmeas a partir de 13 meses de idade e verificou-se maior concentração destes sinais aos 14 (T8) e aos 15 meses (T3), compatível com a atividade ovariana aos 15/16 meses, quando os animais destes grupos tinham folículos antrais e/ou corpos lúteos. Estes resultados permitem concluir que a adição de monensina à alimentação de fêmeas Girolando pré-púberes isolada ou associada com bioestimulação e aplicação de rBST permite homogeneizar a idade à puberdade de animais criados a pasto, reduzindo a idade ao primeiro serviço e parto.

Palavras-chave: estro, fêmeas, manejo, reposição.

\section{ABSTRACT}

To evaluate the effect of three management strategies on ovarian function of Girolando heifers raised in a pasture-based system, 80 females with 10 months of age and weight $169,3 \pm 26,4 \mathrm{~kg}$ were divided in eight groups and submitted by 180 days to the treatments: Control (T1); Biostimulation (T2); Monensin (T3); Recombinant bovine somatotropin (BST) (T4); Biostimulation and Monensin (T5); Biostimulation and BST (T6); Monensin and BST (T7); Biostimulation, Monensin and BST (T8). Oestrus activity was evaluated daily and heifers were weighted every 15 days. Starting from the 15th month of age animals were 
submitted to ovarian evaluation by ultrasound to verify follicles and corpus luteum. Daily weight gain (kg) was 0,52 $\pm 0,02$ (T1); 0,42 $\pm 0,03$ (T2); 0,68 $\pm 0,04$ (T3); 0,43 $\pm 0,03$ (T4); 0,42 $\pm 0,04$ (T5); 0,42 $\pm 0,02$ (T6); 0,70 $\pm 0,04$ (T7) and 0,53 $\pm 0,01$ (T8), with differences $(\mathrm{P}<0,05)$. Weight on the 16th month of age varied from $270 \mathrm{~kg}$ (T2) to $323 \mathrm{~kg}$ (T3), with differences $(P<0,05)$. The signals of oestrus where observed in females starting from the 13th month of age and the higher concentration of these signals being observed at the 14th (T8) and 15th (T3), compatible with the ovarian activity to the 15 months when the animals of these groups had antral follicles or corpus luteum. The present results allow to conclude that the offer of monensin to pre-pubertal Girolando heifers, alone or in association with bioestimulation and BST allows homogenizing the age at puberty in pasture-based systems, reducing the age of the first service and calving.

Key words: female, management, oestrus, reposition.

\section{INTRODUÇÃO}

Em sistemas de produção de bovinos leiteiros, assim como em outros sistemas, a busca pela eficiência na produção e o aumento da produtividade é uma necessidade crescente do produtor para a permanência na atividade. Neste contexto, novilhas de reposição devem receber maior atenção, pois não possuem produção direta na geração de receita (Salles et al., 2001). Assim, o manejo imposto a essa categoria deve aliar 0 ganho de peso ao desencadeamento da puberdade, promovendo maior rapidez na obtenção de novilhas gestantes e posteriormente, no grupo de vacas em produção.

Entretanto, em sistemas extensivos, a produção sazonal de forragem dificulta o rápido e contínuo ganho de peso, e consequentemente, a puberdade em novilhas, pois segundo Ferreira (1993), o início da atividade ovariana é mais dependente do peso corporal do que da idade.

A bioestimulação é uma alternativa para favorecer a redução da idade à puberdade e o aumento no ganho de peso em novilhas pré-púberes (Oliveira et al., 2007). Quadros e Lobato (2004) também observaram maior ciclicidade e maior taxa de prenhez em novilhas acompanhadas de rufião quando comparadas ao grupo controle.

A adição de aditivos na dieta de ruminantes tem sido outra opção para minimizar as consequências da irregularidade na produção de pastagens e do fornecimento de energia no ganho de peso dos animais. A monensina sódica, assim como outros ionóforos, diminui a proporção de bactérias gram positivas e aumenta a de gram negativas, modificando a fermentação ruminal (National Research Council - NRC, 2001). A monensina sódica melhora a conversão alimentar e o ganho de peso médio diário, podendo levar à precocidade reprodutiva dos animais, pois melhora a eficiência do metabolismo energético e nitrogenado no rúmen (Salles et al., 2001). Meinert et al. (1992), em estudo sobre o efeito da monensina no crescimento corporal, no estado corporal e no desempenho reprodutivo, verificaram redução de idade à puberdade em novilhas Holandesas. Apesar disso, os tratamentos com monensina não alteraram significativamente a condição corporal e o ganho de peso.

A somatotropina recombinante bovina (rBST), aplicada em pequenas doses, também pode acelerar o crescimento das novilhas, e, conseqüentemente, na redução da idade ao parto. Segundo Lucy (2000), a rBST pode estimular diretamente a atividade 
ovariana, alterando os padrões de dinâmica folicular.

O objetivo do presente estudo foi avaliar a utilização de três diferentes estratégias de manejo: bioestimulação, adição de monensina sódica na dieta e aplicação de rBST, isoladas ou associadas, sobre o peso, idade e início da puberdade de novilhas Girolando criadas a pasto.

\section{MATERIAL E MÉTODOS}

Foram utilizadas 80 novilhas Girolando, com idade inicial de 10 meses e pertencentes ao mesmo sistema de produção de leite localizado no município de Leopoldo de Bulhões - GO. Os animais com peso médio de $169,3 \pm 26,4 \mathrm{~kg}$ foram mantidos, durante 180 dias, em piquetes de Brachiaria brizantha cv marandu, recebendo $1 \mathrm{~kg}$ de milho triturado/animal/dia, suplemento mineral e resíduo de cervejaria ofertado no cocho ad libitum, sendo este denominado padrão.

As novilhas foram distribuídas aleatoriamente em oito grupos de 10 animais, sendo: T1, controle, manejo padrão; T2, bioestimulação, presença de rufião; T3, monensina, adição de monensina sódica à dieta $(1$ a 1,5 g/animal/dia, de acordo com o crescimento corporal); T4, rBST, aplicação intramuscular de 125 a $250 \mathrm{mg}$ de somatotropina recombinante bovina, em intervalos de 14 dias; T5, bioestimulação e monensina; T6, bioestimulação e rBST; T7, monensina e rBST e T8, bioestimulação, monensina e rBST.

Os animais foram observados diariamente para verificação de sinais de estro e pesados a cada 15 dias. Entre o 15응 e $16^{\circ}$ mês de idade foram realizados exames ultra-sonográficos dos ovários para avaliação da atividade ovariana, determinada pela presença de folículos ovarianos de diâmetro igual ou superior a 5 mm e corpos lúteos.

O delineamento experimental utilizado foi o inteiramente casualizado em um esquema fatorial $2 \times 2 \times 2$ com oito tratamentos e a análise de variância foi realizada utilizando-se o programa WinStat (2006). De acordo com a instabilidade das variáveis estudadas $(\mathrm{CV})$ e o número de tratamentos o teste de média escolhido foi o de Duncan (Sampaio, 2002).

\section{RESULTADOS E DISCUSSÃO}

O ganho de peso médio diário (GMD) variou de 0,42 $\pm 0,03 \mathrm{~kg}$ para T6 a $0,70 \pm 0,05 \mathrm{~kg}$ para T7 (Tabela 1), com diferenças $(P<0,05)$ entre os grupos. $O$ GMD dos animais bioestimulados (T2, T5, T6 e T8) não diferiu do tratamento controle (T1). Oliveira et al. (2007) e Rekwot et al (2001), estudando este efeito em novilhas zebuínas, verificaram que a bioestimulação aumentou o ganho de peso. Embora ainda pouco elucidado, um dos mecanismos pelos quais a bioestimulação atua sobre 0 peso das novilhas é o estímulo sobre o hipotálamo, promovendo 0 aumento na pulsatilidade do Hormônio Liberador das Gonadotrofinas (GnRH) e, conseqüentemente, do Hormônio Luteinizante (LH) que atua sobre os ovários, antecipando as primeiras ovulações e favorecendo 0 efeito anabolizante da progesterona (OLIVEIRA et al., 2007).

O GMD dos animais tratados com monensina isolada e/ou associada a rBST (T3 e T7) foi superior aos demais, concordando com os resultados de Salles et al. (2001). Os animais tratados apenas com monensina (T3) também obtiveram maior peso aos 16 meses, diferindo dos demais tratamentos $(P<0,05)$.

O comportamento de estro foi verificado a partir dos 13 meses de idade, porém de forma irregular. O T5 apresentou maior número de novilhas com comportamento de estro entre o $13^{\circ}$ e o $15^{\circ}$ mês, resultados que concordam com Oliveira et al. (2007) sobre o efeito da bioestimulação na diminuição da idade à puberdade de novilhas. Salles et al. (2001) 
Tabela 1- Média $(\mu)$ e erro padrão (EP) do ganho de peso médio diário (GMD), peso aos 16 meses, comportamento estral e atividade ovariana em novilhas girolando submetidas aos tratamentos com bioestimulação, monensina sódica e rBST, Leopoldo de Bulhões/GO, 2006.

\begin{tabular}{|c|c|c|c|c|c|c|}
\hline \multirow{3}{*}{ Tratamentos } & \multirow{3}{*}{$\mathbf{N}$} & \multirow[t]{2}{*}{ GMD (kg) } & \multirow{2}{*}{$\begin{array}{c}\text { Peso aos } 16 \\
\text { meses }(\mathrm{kg})\end{array}$} & \multicolumn{2}{|c|}{$\begin{array}{c}\text { Comportamento } \\
\text { estral (\%) }\end{array}$} & $\begin{array}{c}\text { Atividade } \\
\text { Ovariana(\%) }\end{array}$ \\
\hline & & & & \multicolumn{3}{|c|}{ Idade em meses } \\
\hline & & $\mu \pm E P$ & $\mu \pm E P$ & 13 & 16 & 15-16 \\
\hline T1 & 10 & $0,52 \pm 0,02^{a, b}$ & $306,1 \pm 14,0^{a}$ & 10 & 30 & 50 \\
\hline $\mathrm{T} 2$ & 10 & $0,43 \pm 0,03^{a, b}$ & $270,0 \pm 12,9^{a}$ & 0 & 20 & 80 \\
\hline T3 & 10 & $0,68 \pm 0,04^{c}$ & $323,1 \pm 15,8^{b}$ & 0 & 70 & 80 \\
\hline T4 & 10 & $0,43 \pm 0,03^{a, b}$ & $287,7 \pm 8,7^{a}$ & 20 & 10 & 90 \\
\hline T5 & 10 & $0,42 \pm 0,05^{\mathrm{a}}$ & $272,6 \pm 15,0^{a}$ & 30 & 50 & 30 \\
\hline T6 & 10 & $0,42 \pm 0,03^{a}$ & $271,5 \pm 12,1^{\mathrm{a}}$ & 10 & 20 & 80 \\
\hline T7 & 10 & $0,70 \pm 0,05^{c}$ & $302,5 \pm 16,5^{\mathrm{a}}$ & 10 & 30 & 70 \\
\hline T8 & 10 & $0,53 \pm 0,02^{b}$ & $303,2 \pm 9,9^{a}$ & 0 & 100 & 50 \\
\hline
\end{tabular}

Valores acompanhados por letras diferentes na mesma coluna indicam diferença $(\mathrm{P}<0,05)$ pelo teste de Duncan.

e Meinert et al. (1992) verificaram a ação da monensina sódica na redução da idade ao início da atividade ovariana. A monensina sódica promove alterações ruminais que favorecem a produção de ácido propiônico e consumo de lactato, resultando na elevação das concentrações séricas de glicose, a qual, segundo Schillo (1992), tem importante papel na modulação dos pulsos de $\mathrm{LH}$.

Aos 15 meses de idade verificou-se, para 0 T3, maior homogeneidade no comportamento de estro, ou seja, maior número de fêmeas em estro durante o mesmo período, mas aos 16 meses, o grupo mais homogêneo foi T8. Apesar disso, o T3, aos 15/16 meses, apresentou melhor correspondência entre o número de animais que apresentaram sinais de estro e a atividade ovariana, com $70 \%$ das novilhas com comportamento estral e $80 \%$ das novilhas com presença de estruturas ovarianas, no mesmo período.

A rBST tem atividade biológica similar à somatotropina natural, controlando diversos aspectos do crescimento animal e do metabolismo de nutrientes, e tem sido utilizada em vacas leiteiras para aumentar a produção, apresentando ainda atividade indutora na produção do fator de crescimento semelhante à insulina - tipo 1
(IGF-1), tanto no fígado quanto no ovário (Lucy, 2000).

O IGF-1 atua em sinergismo com as gonadotrofinas, estimulando o crescimento e a esteroidogênese das células ovarianas. Ao comparar o número de novilhas com comportamento estral, folículos antrais e corpos lúteos dos grupos tratados com monensina e rBST, fica evidenciado o efeito glicogênico da primeira e indutor da produção de fatores de crescimento da rBST.

\section{CONCLUSÕES}

Os resultados permitem concluir que a adição de monensina sódica à alimentação de novilhas Girolando prépúberes isolada ou associada com bioestimulação e aplicação de rBST possibilita homogeinização da idade à puberdade de animais criados a pasto, reduzindo a idade ao primeiro serviço e conseqüentemente ao parto e pode ser uma alternativa para o manejo de novilhas de reposição.

\section{REFERÊNCIAS}

FERREIRA, A.D. Nutrição e atividade ovariana em bovinos: uma revisão. Pesquisa Agropecuária Brasileira, v.28, n.9, p.1077-1093, 1993. 
LUCY, M.C. Regulation of ovarian follicular growth by somatotropin and insulin-like growth factors in cattle. Journal of Dairy Science, v.83, n.7, p.16351647, 2000.

MEINERT, R.A; YANG, C.M.J.; HEINRICHS, A.J; VARGA, G.A. Effect of monensin on growth, reproductive perfomance, and estimated body composition in Holstein heifers. Journal of Dairy Science, v.75, n.1, p.257-261, 1992.

NATIONAL RESEARCH COUNCIL. Unique aspects of dairy cattle nutrition. In: NATIONAL RESEARCH COUNCIL. Nutrient Requirements of Dairy Cattle. 7.ed. Washington: National Academy Press, 2001, p.184-213.

OLIVEIRA, C.M.G.; OLIVEIRA FILHO, B.D.; GAMBARINI, M.L.;VIU, M.A.O.; LOPES, D.T. Effects of biostimulation and nutricional supllementation on pubertal age and pregnancy rates of Nellore heifers (Bos taurus indicus) in a tropical environment. Animal Reproduction Science, 2007. [no prelo]

QUADROS, S.A.F.; LOBATO, J.F.P. Bioestimulação e comportamento reprodutivo de novilhas de corte. Revista Brasileira de Zootecnia, v.33, n.3, p.679683, 2004.

REKWOT, P.I.; OGWU, D.; OYEDIPE, E. O.; SEKONI, V.O. The role of pheromones and biostimlation in animal reproduction. Animal Reproduction Science, v.65, n.3-4, p.157-170, 2001.

SALLES, M.S.V.; ZANETTI, M.A.; CONTI, R.M.C; LIMA, C.G. Efeitos da monensina no desempenho de bezerras leiteiras em crescimento. Revista Brasileira de Zootecnia, v.30, n.4, p.1293-1298, 2001.

SAMPAIO, I.B.M. Estatística Aplicada à Experimentação Animal. 2.ed. Belo Horizonte: Fundação de Estudo e Pequisa em Medicina Veterinária e Zootecnia, 2002, p. 265.

SCHILLO, K.K. Effects of dietary energy on control of luteinizing hormone secretion in cattle and shepp. Journal of Animal Science, v.70, n.4, p.1271-1282, 1992. 